\title{
Travelling gradients in interacting morphogen systems
}

\author{
R.E. Baker ${ }^{a, *}$ P.K. Maini ${ }^{a, b}$ \\ ${ }^{a}$ Centre for Mathematical Biology, Mathematical Institute, University of Oxford, 24-29 St. Giles', Oxford OX1 3LB, UK \\ ${ }^{\mathrm{b}}$ Oxford Centre for Integrative Systems Biology, Department of Biochemistry, South Parks Road, Oxford OXI 3QU, UK
}

Received 11 August 2006; received in revised form 21 November 2006; accepted 23 January 2007

Available online 8 February 2007

\begin{abstract}
Morphogen gradients are well known to play several important roles in development; however the mechanisms underlying the formation and maintenance of these gradients are often not well understood. In this work, we investigate whether the presence of a secondary morphogen can increase the robustness of the primary morphogen gradient to perturbation, thereby providing a more stable mechanism for development. We base our model around the interactions of Fibroblast Growth Factor 8 and retinoic acid, which have been shown to act as morphogens in many developmental systems. In particular, we investigate the formation of opposing gradients of these morphogens along the antero-posterior axis of vertebrate embryos, thereby controlling temporal and spatial aspects of axis segmentation and neuronal differentiation.

(C) 2007 Elsevier Inc. All rights reserved.
\end{abstract}

PACS: 87.17.Aa; 87.18.La; 87.18.Hf; 02.60.Lj

Keywords: Morphogen gradients; Mathematical modelling; FGF8; Retinoic acid; Segmentation

\section{Introduction}

Morphogens are secreted signalling molecules that provide spatial information during embryonic development, typically via concentration gradients. Their role is to organise fields of cells;

\footnotetext{
${ }^{*}$ Corresponding author. Tel.: +44 1865 283889; fax: +44 1865283882.

E-mail addresses: ruth.baker@maths.ox.ac.uk (R.E. Baker), maini@maths.ox.ac.uk (P.K. Maini).

URLs: http://www.maths.ox.ac.uk/ baker (R.E. Baker), http://www.maths.ox.ac.uk/ maini (P.K. Maini).
} 
determining cell arrangement and/or fate via differing responses to threshold levels of morphogen concentration $[1,2]$. As such, this type of patterning provides a highly efficient mechanism for creating complex patterns in the embryo and there are numerous examples of the roles of morphogens in developmental systems. For example: neuronal subtype specification in the developing central nervous system (CNS) is controlled by a gradient of Sonic Hedgehog (Shh) [3]; formation of the dorsal pattern in Drosophila is controlled by gradients of Screw ( $\mathrm{Scw}$ ) and Decapentaplegic (Dpp) [4]; antero-posterior (AP) patterning in the chick limb is controlled by a gradient of Shh arising from a polarising region at the posterior edge of the limb bud [5].

Typical morphogen gradient models assume that a morphogen produced at a localised source diffuses across a target field to set up an extracellular gradient. Cells then determine their position within the field by interpretation of the morphogen gradient, activating specific programs of differentiation at discrete morphogen thresholds [5]. A lesser cited way in which morphogens provide information for differentiation is via the action of a gradient which travels across the target field over time [6,7]. In these models, there is ordinarily a single morphogen threshold and progression of the threshold across the field acts to control the time of onset of differentiation rather than to select the program of differentiation: it is generally assumed that the program itself is already decided. In addition the picture has been complicated by the discovery that in some settings different morphogens act in combination with one another to set thresholds for development: for example, recent studies have shown that some developmental systems require two morphogens which mutually inhibit each other [8].

To understand the mechanisms underlying the formation and maintenance of any gradient we should ideally know the exact identity of the morphogens involved, rates of morphogen release, diffusion and decay, etc. For most morphogens, these aspects are not very well understood and this is where mathematical modelling can play an important role. It may be used to investigate the feedback mechanisms underlying gradient formation, for example, how the activity of a primary morphogen may be partly controlled by the action of secondary morphogens, or the range of influence of a gradient. In addition, mathematical modelling can be used to make predictions and experimentally testable hypotheses which further understanding in the area.

However, as important as the study of the above mechanisms, is the study of gradient robustness: biological systems are subject to stochastic effects from a variety of sources. There are many ways to define 'robustness' but one which encompasses a wide range of meanings is 'a measure of how sensitive the output of a system is to perturbation'. In the context of a mathematical model, perturbation generally means variation in model parameters or initial conditions. We note that parameters may be changed in a 'step-wise manner', that is, a parameter value may be changed from one value to another between different simulations, or in a 'noisy' manner, that is, a parameter may make small, random departures from its chosen value at each time step of a particular simulation. The former may be valid when considering, for example, the effect of temperature change on a system [9] - this may affect production and decay rates. On the other hand, if only one or two gene copies are responsible for mRNA transcription in a system, there is bound to be variability in this rate from one time interval to another - this is where the latter line of investigation becomes important.

There has been much theoretical and experimental investigation of morphogen gradients (see, for theoretical models, $[10,11]$ ), including the mechanisms via which a robust gradient may be achieved $[9,12,13]$. In each case static morphogen gradients are studied, and robustness is defined 
as the ability of the system to adjust to changes in parameter values, such that the position of a threshold in morphogen concentration remains virtually unaltered. Bollenbach and co-workers show that robustness of a single morphogen gradient may arise as a result morphogen transport via a series of transcytotic events [12], whilst Houchmandzadeh and co-workers and AegerterWilmsen and co-workers consider the addition of a secondary morphogen in order to generate a robust response to domain scaling $[9,13]$. One other study investigates stationary gradients of Bicoid in the Drosophila embryo; postulating the existence of a secondary morphogen to ensure robust activation of downstream genes such as hunchback [14].

Development is a precise process, yet all biochemical processes are prone to variation: our hypothesis is that the addition of secondary morphogens could provide a more stable mechanism for the formation of travelling morphogen gradients. Theoretical investigation of a system consisting of two negatively regulating morphogens permits us to address this hypothesis and investigate whether this process leads to more robustly controlled travelling gradients.

\subsection{Aims and outline}

In this article we will be interested in a scenario in which the interactions of two negatively regulating morphogens lead to gradients which travel across a developmental field, activating, as they travel, a program of further differentiation. We will base our study on the interactions of Fibroblast Growth Factor 8 (FGF8) and retinoic acid (RA) seen to occur along the antero-posterior (AP) axis of vertebrate embryos: the expression gradients formed have been shown to be important in segmentation of the vertebrate axis and also in neuronal differentiation $[8,15]$. We begin by outlining our context for investigation in more detail: highlighting the salient factors leading to the formation of gradients in FGF8 and RA along the AP axis and their role in development. We then construct a general model which can be used to investigate the system, presenting both analytical results and numerical simulations. Before carrying out extensive analysis of this system, we consider the case in which the secondary morphogen is removed: this case is much easier to investigate and provides important insight into the general model. Finally we discuss our results, avenues for future exploration and the implications for such mechanisms in development.

\section{The developmental context}

In this article, the specific developmental scenario which we will model is the interplay of two opposing morphogens, FGF8 and RA, involved in the progressive maturation of the vertebrate embryo along its AP axis [8,15]. Although RA and FGF8 have long since been shown to act as morphogens in many different developmental contexts [16-23] it is only recent studies that have shown that opposing gradients of RA and FGF8 travelling down the embryonic AP axis are essential for segmentation of the vertebrate AP axis into somites and for coordinated neuronal differentiation and ventral patterning [6,24-26]. It is this phenomenon in which we will be interested in this article.

Somites, the precursors of the vertebrae, ribs and associated muscles of the trunk, are derived from two parallel bands of tissue known as the pre-somitic mesoderm (PSM) that lie on either side of the midline of the embryo. At regular time intervals (every $90 \mathrm{~min}$ in the chick [27]), a group of cells at the anterior end of the PSM undergo changes in their adhesive and migratory properties 
and coalesce to form an epithelial block of cells known as a somite [28]. Somites form in strict AP sequence [29-31] and this budding of cells from the anterior part of the PSM compensates for the addition of cells at the posterior end of the PSM as the body axis lengthens. In this way, the PSM travels down the AP axis, remaining approximately constant in length throughout the process of segmentation, and a wave of cell determination appears to sweep along the AP axis behind the PSM leaving somites in its wake [32,33].

The most commonly accepted mechanism for somitogenesis is the 'Clock and Wavefront' model, first postulated by Cooke and Zeeman [34]. Recent experimental observations have shown that FGF8 provides a likely candidate for the wavefront [6], and there is wide evidence for the segmentation clock [35]. With the discovery of these factors, Pourquié and co-workers have proposed a revised version of the 'Clock and Wavefront' model $[6,36,37]$. They hypothesise that there is some interaction between the wavefront of FGF8 and the segmentation clock in the PSM that acts to gate cells into potential somites. FGF8 is expressed at a high level in the posterior PSM, and the gradient of FGF8 regresses along the AP axis as the embryo develops, so that cells gradually go from regions of high to low FGF8 signalling. For a cell at a particular point, they assume that competence to segment will only be achieved once the FGF8 gradient has regressed far enough along the AP axis and FGF8 signalling has decreased below a certain threshold.

It is postulated that the FGF8 gradient arises via a feedback loop with RA: FGF8 may either down-regulate RA production via repression of the enzyme Raldh2, or it may increase the rate of RA decay; in turn RA may either down-regulate FGF8 production by restricting $f g f 8$ mRNA transcription or it may accelerate the rate of decay via the MPK3 pathway [24].

\subsection{Objectives for the model}

We will be interested in constructing a model consisting of non-linear, coupled partial differential equations: one equation describing the dynamics of FGF8 and another the dynamics of RA. We will explore the system in one spatial dimension, along the AP axis, and look for travelling wave solutions, which correspond to gradients of FGF8 and RA moving along the AP axis. In the scenario under consideration, FGF8 expression is highest in the posterior end of the embryo (denoted here by letting $x \rightarrow \infty$ ) and this gradient regresses along the axis in a posterior direction as development proceeds (i.e., the interface between high and low FGF8 signalling travels in a positive $x$ direction).

Before beginning our investigation, we outline a definition of robustness for this system. First, we note that in order for the model to be able to exhibit travelling waves of expression which are robust to small variation in FGF8/RA concentrations ahead and behind the travelling wavefront, we need to restrict ourselves to waves which connect steady states of the form $\left(f^{*}, 0\right)$ and $\left(0, a^{*}\right)$ which are both linearly stable. Our reasoning for this goes as follows: supposing we have a scenario in which a travelling wave connects a state $\left(f^{*}, 0\right)$, which is linearly unstable, to a state $\left(0, a^{*}\right)$, which is linearly stable. It is possible that small perturbations in either $f$ or $a$ could be applied to the system due to slight changes in morphogen production, decay or other, possibly external, factors. If they occur in the region of the axis not yet reached by the wavefront (i.e., as $x \rightarrow \infty$ ) then these perturbations will grow since the state is unstable, and it is possible that the gradient could be destroyed. Hence in this work, we will consider waves of the above form and explore the effects upon these travelling waves of varying the type and strength of interactions between FGF8 and RA. 
Second, we would like to explore the response of the system to 'step-wise' parameter changes and we will characterise this response in terms of the speed (and shape) of the travelling wavefront. Our primary interest will be to explore the range of parameters for which the wavefront travels in a positive $x$ direction (for the reasons described earlier); an increase in robustness would be indicated by a widening of this parameter space. However, the actual speed of wavefront progression is also important. We will also infer from our results which types of morphogen interaction lead to a more tightly controlled wave speed.

\section{Mathematical model}

As discussed in the opening sections, we will investigate the negative feedback interactions between two morphogens, FGF8 and RA, which lead them to form gradients in their expression levels which travel along the AP axis of vertebrate embryos. The ability of cells to differentiate is controlled by the time at which FGF8 levels reach some critical threshold: in this way, the program of development is controlled temporally and spatially by the morphogen. The type and strength of interactions between the morphogens will control the shape of the gradients and the rate at which they travel along the AP axis.

We consider an infinite, one-dimensional domain, the $x$ axis (corresponding to the AP axis with the tail of the embryo lying towards $x=\infty$ ), and we will assume that the concentrations of the morphogens are bounded as $x \rightarrow \pm \infty$. Our two morphogens will be labelled $\boldsymbol{F}$ and $\boldsymbol{A}$ (after FG8 and RA) and we suppose that their interactions are limited to those depicted in Fig. 1 (although all such interactions may not necessarily occur). The most general mathematical model describing this system can be written as

$$
\begin{aligned}
& \frac{\partial F}{\partial t}=p_{F}(F, A)-d_{F}(F, A)+D_{F} \frac{\partial^{2} F}{\partial x^{2}}, \\
& \frac{\partial A}{\partial t}=p_{A}(F, A)-d_{A}(F, A)+D_{A} \frac{\partial^{2} A}{\partial x^{2}}
\end{aligned}
$$

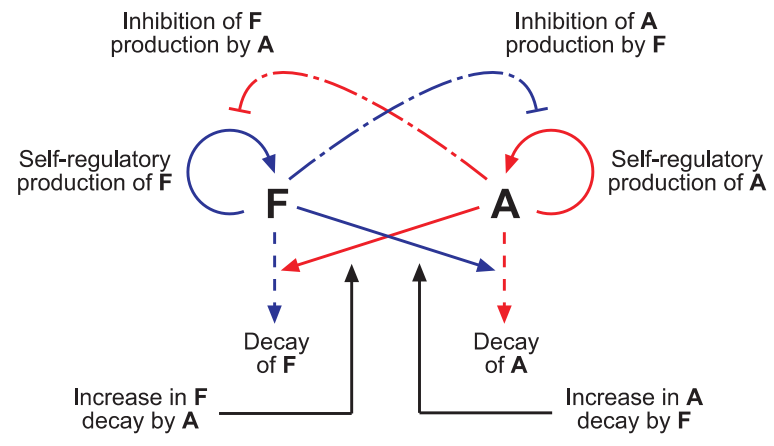

Fig. 1. A graphical representation of the interactions taking place between morphogens $\boldsymbol{F}$ and $\boldsymbol{A}$. Morphogen $\boldsymbol{F}$ undergoes self-regulatory production, which may be suppressed by the presence of $\boldsymbol{A}$, and linear decay, which may be augmented by the presence of $\boldsymbol{A}$. Similarly for morphogen $\boldsymbol{A}$. Actions of morphogen $\boldsymbol{F}$ are represented in blue, and those of $\boldsymbol{A}$ in red. (For interpretation of the references to colour in this figure legend, the reader is referred to the web version of this article.) 
where $F$ and $A$ represent, respectively, concentrations of morphogen $\boldsymbol{F}$ and $\boldsymbol{A} . p_{F}(F, A), p_{A}(F, A)$ represent production of $\boldsymbol{F}$ and $\boldsymbol{A}$, respectively, and $d_{F}(F, A), d_{A}(F, A)$ decay of $\boldsymbol{F}$ and $\boldsymbol{A}$, respectively. $D_{F}$ and $D_{A}$ are the respective diffusion rates of $\boldsymbol{F}$ and $\boldsymbol{A}$ and we assume, for the sake of simplicity, that spreading of the morphogens takes places solely by diffusion [38].

In order for the system to display the behaviour shown in Fig. 1 these functions must have the following properties:

$$
\begin{aligned}
& \text { (i) } \frac{\partial p_{F}}{\partial A} \leqslant 0, \frac{\partial p_{F}}{\partial F} \geqslant 0, \frac{\partial d_{F}}{\partial F} \geqslant 0 \text { and } \frac{\partial d_{F}}{\partial A} \geqslant 0 ; \\
& \text { (ii) } \frac{\partial p_{A}}{\partial F} \leqslant 0, \frac{\partial p_{A}}{\partial A} \geqslant 0, \frac{\partial d_{A}}{\partial A} \geqslant 0 \text { and } \frac{\partial d_{A}}{\partial F} \geqslant 0 .
\end{aligned}
$$

The first and fourth restrictions for $\boldsymbol{F}$ ensure that $\boldsymbol{A}$ is able to either down-regulate $\boldsymbol{F}$ production or upregulate $\boldsymbol{F}$ decay (or both), the second ensures that $\boldsymbol{F}$ is self-activating and the third simply that the rate of $\boldsymbol{F}$ decay increases with increasing $\boldsymbol{F}$. The second set of requirements ensures similar behaviour for $\boldsymbol{A}$. In order to have travelling waves which are robust to external perturbations, we also require the system to have a set of spatially uniform steady states which includes at least one stable state each of the form $(F, A)=\left(F^{*}, 0\right),\left(0, A^{*}\right)$.

In order to satisfy these requirements we choose to investigate the system:

$$
\begin{aligned}
& \frac{\partial F}{\partial t}=\frac{r_{F} F^{n_{1}}}{\mu_{F}^{n_{1}}+F^{n_{1}}} \frac{\lambda_{F}^{n_{2}}}{\lambda_{F}^{n_{2}}+A^{n_{2}}}-\left(\eta_{F}+\frac{s_{F} A^{n_{3}}}{\beta_{F}^{n_{3}}+A^{n_{3}}}\right) F+D_{F} \frac{\partial^{2} F}{\partial x^{2}}, \\
& \frac{\partial A}{\partial t}=\frac{r_{A} A^{n_{4}}}{\mu_{A}^{n_{4}}+A^{n_{4}}} \frac{\lambda_{A}^{n_{5}}}{\lambda_{A}^{n_{5}}+F^{n_{5}}}-\left(\eta_{A}+\frac{s_{A} F^{n_{6}}}{\beta_{A}^{n_{6}}+F^{n_{6}}}\right) A+D_{A} \frac{\partial^{2} A}{\partial x^{2}},
\end{aligned}
$$

where the $n_{i}(i=1, \ldots, 6)$ are chosen to be integers greater than unity and the remaining parameters are positive constants. Here $\boldsymbol{F}$ production is self-regulatory, with maximal production rate $r_{F}$, and the rate of $\boldsymbol{F}$ production is decreased by the presence of $\boldsymbol{A}$. In the absence of $\boldsymbol{A}, \boldsymbol{F}$ decays linearly but the decay rate is increased by the presence of $\boldsymbol{A}$, up to a maximal rate of $\eta_{F}+s_{F}$. Similar statements are true for $\boldsymbol{A}$. We also note that this model allows us to consider the effects of removing either morphogen: in Eq. (3) when we remove $\boldsymbol{A}$ the first fraction involving $\boldsymbol{A}$ becomes unity and the second becomes zero. Similarly for Eq. (4) and removing $\boldsymbol{F}$.

We note that, in this model, we are implicitly assuming that both morphogens are present in high enough concentrations to average out the fluctuations in production/decay rates which arise on the individual molecule level.

\subsection{Non-dimensionalisation}

The system given by Eqs. (3) and (4) can be non-dimensionalised to give

$$
\frac{\partial f}{\partial \tau}=\frac{r_{f} f^{n_{1}}}{1+f^{n_{1}}} \frac{\lambda_{f}^{n_{2}}}{\lambda_{f}^{n_{2}}+a^{n_{2}}}-\left(\eta_{f}+\frac{s_{f} a^{n_{3}}}{\beta_{f}^{n_{3}}+a^{n_{3}}}\right) f+D \frac{\partial^{2} f}{\partial \xi^{2}},
$$




$$
\frac{\partial a}{\partial \tau}=\frac{r_{a} a^{n_{4}}}{1+a^{n_{4}}} \frac{\lambda_{a}^{n_{5}}}{\lambda_{a}^{n_{5}}+f^{n_{5}}}-\left(\eta_{a}+\frac{s_{a} f^{n_{6}}}{\beta_{a}^{n_{6}}+f^{n_{6}}}\right) a+\frac{\partial^{2} a}{\partial \xi^{2}},
$$

where

$$
\begin{aligned}
& F=\mu_{F} f, \quad A=\mu_{A} a, \quad t=[T] \tau, \quad x=\sqrt{D_{A}[T]} \xi, \quad D=\frac{D_{F}}{D_{A}}, \\
& \lambda_{f}=\frac{\lambda_{F}}{\mu_{A}}, \quad \lambda_{a}=\frac{\lambda_{A}}{\mu_{F}}, \quad \beta_{f}=\frac{\beta_{F}}{\mu_{F}}, \quad \beta_{a}=\frac{\beta_{A}}{\mu_{A}}, \quad r_{f}=\frac{r_{F}[T]}{\mu_{f}}, \\
& r_{a}=\frac{r_{A}[T]}{\mu_{a}}, \quad \eta_{f}=\eta_{F}[T], \quad \eta_{a}=\eta_{A}[T], \quad s_{f}=s_{F}[T], \quad s_{a}=s_{A}[T] .
\end{aligned}
$$

Usually [T] is chosen to eliminate one of $r_{f}$ or $r_{a}$ but since we wish to compare the effects of $\boldsymbol{F}$ upon $\boldsymbol{A}$ and vice versa we leave the choice of $[T]$ open.

\subsection{Numerical simulation}

The system described by Eqs. (5) and (6) must be solved numerically and the results of numerical simulation for a particular set of parameter values are shown in Fig. 2. The system is solved using the MATLAB solver pdepe with the infinite domain approximated by a finite domain with zero flux boundary conditions for both morphogens. The initial conditions are such that $\boldsymbol{F}$ is in a non-zero, stable steady state for $\xi<0$ and zero on the remainder of the domain, and vice versa for $\boldsymbol{A}$. For the set of parameter values chosen, the waves move in a positive direction over time, indicating a regressing gradient of morphogen $\boldsymbol{F}$ and an advancing gradient of $\boldsymbol{A}$. After an initial transient period, the wavefront assumes constant shape and moves along the axis with constant speed. In a biological context, this could be equivalent to conferring the ability to differentiate,
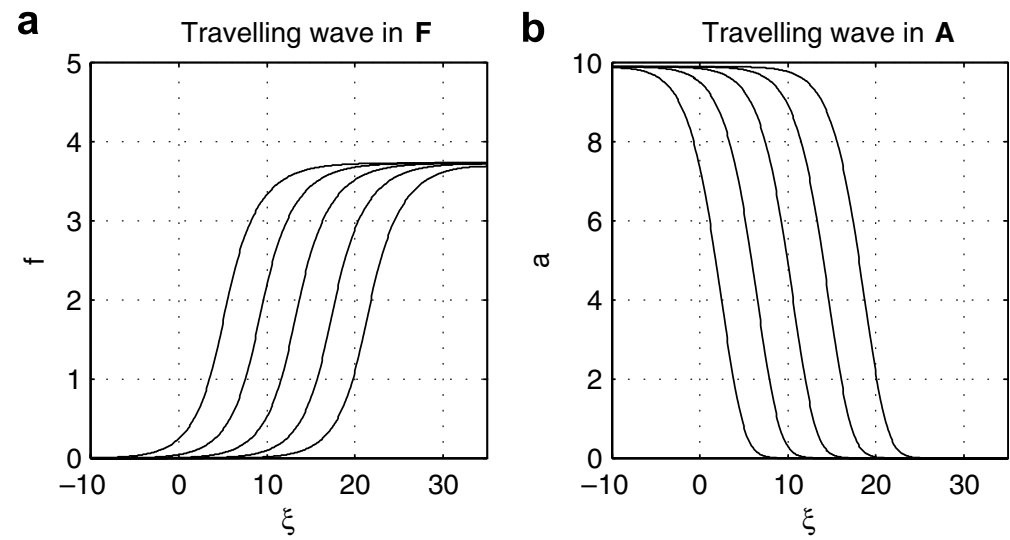

Fig. 2. Numerical solution of the model given by Eqs. (5) and (6). (a) The region of high $\boldsymbol{F}$ concentration regresses along the axis with time. (b) At the same time the region of high $\boldsymbol{A}$ concentration advances. Plots are at $\tau=10,20,30$, 40, 50. Parameters are as follows: $r_{f}=2.0, r_{a}=5.0, \eta_{f}=0.5, \eta_{a}=0.5, \lambda_{f}=1.0, \lambda_{a}=1.0, s_{f}=0.5, s_{a}=0.5, \beta_{f}=1.0$, $\beta_{a}=1.0, D=5.0$ and $n_{i}=2$ for $i=1, \ldots, 6$. 
or initiating a developmental program at a constant rate upon cells which are evenly distributed along some developmental axis.

We are predominantly interested in how the presence of a secondary morphogen, which here we will choose to be $\boldsymbol{A}$, influences the shape and speed of a travelling wave of primary morphogen (here $\boldsymbol{F}$ ). The system described above is complex, partly since it is designed to capture both possibilities for regulation: suppression of morphogen production and increase in morphogen decay. It is therefore difficult to gain much insight into the model without the support of insight gained from the case in which we have a single morphogen, and so it is to this case we turn first.

\section{The case in which $A$ is removed}

The purpose here is to investigate the 'knockout' case in which the secondary morphogen, $\boldsymbol{A}$, is removed. This is a simplified form of the system, which is easier to tackle analytically, and which will give important insights into the more general case. The resulting non-dimensional equation that must be investigated is

$$
\frac{\partial f}{\partial \tau}=\frac{r_{f} f^{n_{1}}}{1+f^{n_{1}}}-\eta_{f} f+D \frac{\partial^{2} f}{\partial \xi^{2}} .
$$

The non-zero steady states satisfy the equation

$$
s\left(f ; b_{f}, n_{1}\right)=b_{f}\left(1+f^{n_{1}}\right)-f^{n_{1}-1}=0,
$$

where $b_{f}=\eta_{f} / r_{f}$. Depending on the value of $b_{f}$ there will be one or three steady states, $f=0, f_{ \pm}^{*}$, and we can determine the critical value of $b_{f}$ by a graphical argument (see Fig. 3). First we calculate the points at which the derivative of $s\left(f ; b_{f}, n_{1}\right)=0$ : these can be found to be

$$
f=0 \quad \text { for } \quad n_{1}=3,4, \ldots \quad \text { and } \quad f=\bar{f}=\frac{n_{1}-1}{b_{f} n_{1}} \quad \text { for } \quad n_{1}=2,3, \ldots
$$

Considering the form of the null cline for $f>0$ (see Fig. 3) we see that in order for there to be two non-zero steady states we must have $s\left(\bar{f} ; b_{f}, n_{1}\right)<0$ which occurs when

$$
b_{f}<b_{f}^{\text {crit }_{u}}={\frac{\left(n_{1}-1\right)^{\left(n_{1}-1\right) / n_{1}}}{n_{1}}} .
$$

We can also determine the linear stability of these steady states graphically and we see that $f=0$ is stable whilst (when they exist) $f_{-}^{*}$ is unstable and $f_{+}^{*}$ is stable.

\subsection{Travelling waves}

Using the standard travelling wave ansatz $z=\xi-c \tau$, Eq. (10) becomes

$$
D f^{\prime \prime}+c f^{\prime}+\frac{r_{f} f^{n_{1}}}{1+f^{n_{1}}}-\eta_{f} f=0,
$$



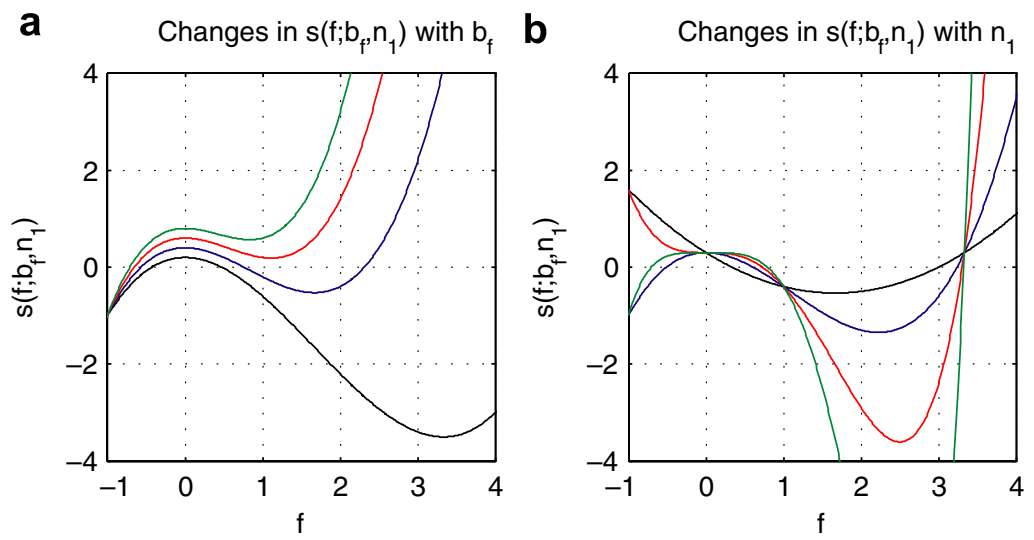

Fig. 3. The 'knockout' null clines given by Eq. (11). (a) The change in the null clines with $b_{f}$ : as $b_{f}$ is increased linearly from 0.2 to 0.8 the null cline goes from the black to the green line. (b) The change in the null cline with $n_{1}$ : as $n_{1}$ is increased linearly from 2 to 5 the null cline goes from the black to the green line. Parameters are as follows: (a) $n_{1}=3$; (b) $b_{f}=0.3$. (For interpretation of the references to colour in this figure legend, the reader is referred to the web version of this article.)

where $^{\prime}=\mathrm{d} / \mathrm{d} z$. Letting $f^{\prime}=g$ we can determine the stabilities of the three steady states in the $\left(f, f^{\prime}\right)$ phase plane: $(0,0)$ is a saddle point; $\left(f_{-}^{*}, 0\right)$ is a stable node or spiral; $\left(f_{+}^{*}, 0\right)$ is a saddle point. In this case, there are three possibilities for travelling waves: the first connects $(0,0)$ to $\left(f_{-}^{*}, 0\right)$; the second connects $\left(f_{+}^{*}, 0\right)$ to $\left(f_{-}^{*}, 0\right)$; and the third connects $(0,0)$ to $\left(f_{+}^{*}, 0\right)$. For reasons discussed earlier, we will be interested in the third case, for which (by continuity arguments [39,40]) there is a unique wave speed, $c$.

Specifying $f(\infty)=f_{+}^{*}$ and $f(-\infty)=0$, so that $\boldsymbol{F}$ is at its upper steady state towards the tail of the embryo, we can multiply Eq. (14) by $f^{\prime}$ and integrate with respect to $z$ to obtain the following expression for the wave speed:

$$
c=-\int_{0}^{f_{+}^{*}}\left[\frac{r_{f} f^{n_{1}}}{1+f^{n_{1}}}-\eta_{f} f\right] \mathrm{d} f / \int_{-\infty}^{\infty}\left(f^{\prime}\right)^{2} \mathrm{~d} z .
$$

Hence the sign of the wave speed is determined by the sign of the numerator integral of Eq. (15), which we will denote as $I=I\left(b_{f}, n_{1}\right)$. For a regressing gradient of $\boldsymbol{F}$ along the axis, as in Fig. 2, we require $c>0$ : whether this actually occurs or not is dependent on the parameter $b_{f}$. Fig. 4 shows how the wave speed depends on $b_{f}$ and $n_{1}$. As $b_{f}$ increases (so that the decay rate increases relative to the maximal production rate) the wave is more likely to travel in a negative direction, whilst increasing the Hill function coefficient is more likely to make the wave travel in a positive direction. The latter suggests that a more defined 'on-off switch' of $\boldsymbol{F}$ production and a smaller morphogen decay rate relative to production rate are more likely to force the wave to travel in a positive direction. These results will be investigated more thoroughly in the following sections.

For each value of $n_{1}$ there will be a corresponding critical value of $b_{f}$ such that $I=0$ : this can be seen by considering the $f$ null cline (see Fig. 3 and consider the changes in the null cline as $b_{f}$ changes). The critical value of $b_{f}$ may be determined analytically, but apart from a couple of limiting cases (specifically $n_{1}=2$ and $n_{1} \rightarrow \infty$ ) the integrals are tedious to calculate (being composed 


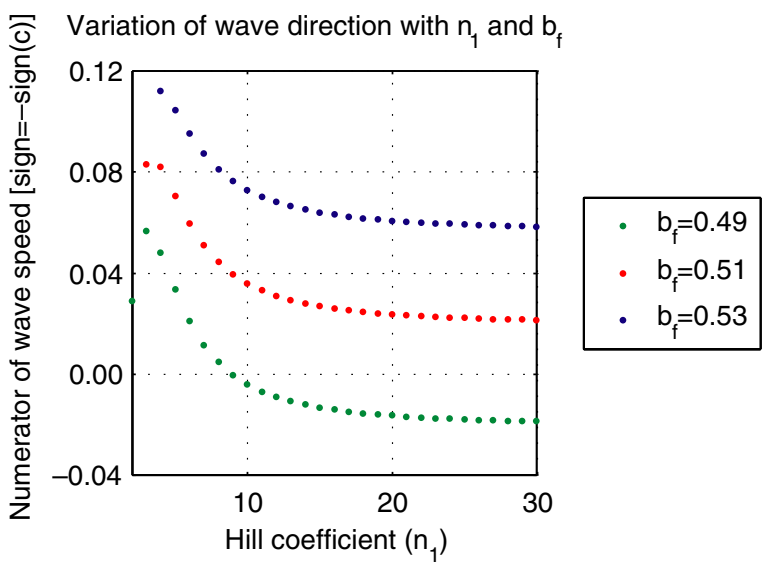

Fig. 4. Variation in the direction of wave progression with $n_{1}$ and $b_{f}$. The sign of the numerator integral (vertical axis) is opposite to the sign of the wave speed: for each value of $b_{f}$ and $n_{1}$, the wave moves in a positive direction when the point lies below zero. The graph shows that if initially the wave moves in a negative direction, increasing $n_{1}$ may force it to move in a positive direction. The graph also suggests that a decrease in $b_{f}$ is more likely to result in a wave travelling in a positive direction. Note that as $b_{f}$ lies above $b_{f}^{\text {crit }}{ }_{\mu}$ for some smaller values of $n_{1}$, no gradient exists.

of Hypergeometric functions). In the next couple of sections we investigate these limiting cases before turning our attention to numerical calculation of the remainder of the cases.

\subsubsection{The case in which $n_{1}=2$}

In this case, we have

$$
\frac{\partial f}{\partial \tau}=\frac{r_{f} f^{2}}{1+f^{2}}-\eta_{f} f+D \frac{\partial^{2} f}{\partial \xi^{2}},
$$

which, for $b_{f}<1 / 2$, has spatially uniform steady states given by $f=0, f_{ \pm}^{*}$ where

$$
f_{ \pm}^{*}=\frac{r_{f}}{2 \eta_{f}}\left[1 \pm \sqrt{1-\left(\frac{\eta_{f}}{2 r_{f}}\right)^{2}}\right]=\frac{1}{2 b_{f}}\left[1 \pm \sqrt{1-4 b_{f}^{2}}\right] .
$$

Evaluating $I\left(b_{f}, 2\right)$, we see that the wave speed will be positive so long as the following constraint is satisfied by $b_{f}$.

$$
\Gamma\left(b_{f}\right)=\frac{1}{4 b_{f}}\left[1+2 b_{f}^{2}+\sqrt{1-4 b_{f}^{2}}\right]-\arctan \left[\frac{1}{2 b_{f}}\left(1+\sqrt{1-4 b_{f}^{2}}\right)\right]<0 .
$$

The function $\Gamma\left(b_{f}\right)$ is plotted in Fig. 5 and the critical value of $b_{f}$ for which the wave speed is equal to zero can be estimated numerically to be $b_{f}^{\text {crit }} \approx 0.4598$. Since the two non-zero steady states, $f_{ \pm}^{*}$, only exist for $b_{f}<1 / 2$ we note that there is only a very small region of $b_{f}$ for which the wave travels in a positive direction. This suggests that a mechanism for gradient formation which obeys the kinetics of Eq. (16), i.e., one in which there is a single morphogen which has Hill function production kinetics with Hill coefficient equal to two, would not be robust to external perturbations and hence would be likely to lead to developmental anomalies. For example, small changes in $\eta_{f}$ or $r_{f}$ 


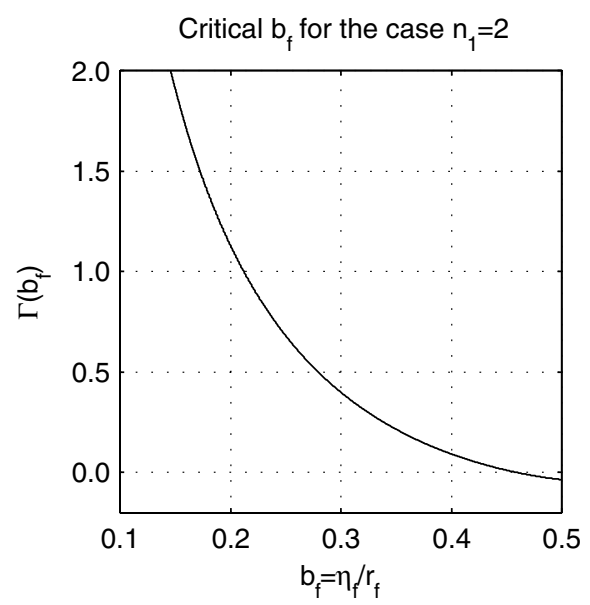

Fig. 5. The change in $\Gamma$ as $b_{f}$ is increased. We see that at $b_{f} \approx 0.4598$ the wave direction changes from negative to positive. Since non-zero steady states only exist for $b_{f}<1 / 2$ this leaves only a small parameter window in $b_{f}$ for waves travelling in a positive direction.

could easily reverse the direction in which the wave travels, thereby disturbing the normal pattern of cell differentiation.

\subsubsection{The case in which $n_{1} \rightarrow \infty$}

Letting $n_{1} \rightarrow \infty$ the production term for $f$ tends towards a Heaviside function centred at $f=1$. In this case, the non-zero steady state exists for $b_{f}<b_{f}^{\text {critu }}=1$ and

$$
\operatorname{sign}\left[I\left(b_{f}, \infty\right)\right]=-\operatorname{sign}\left[\frac{r_{f}^{2}}{2 \eta_{f}} \eta_{f}\left(1-2 b_{f}\right)\right],
$$

so that the wave moves in a positive direction for $b_{f}>b_{f}^{\text {crit }}=1 / 2$. It is interesting to note that the region of $b_{f}$-space for which the wave moves in a positive direction is significantly greater than that for $n_{1}=2$. This suggests that increasing $n_{1}$ increases the robustness of the model, in the sense that it widens the parameter space over which the wave travels in a positive $x$ direction.

\subsubsection{The remaining cases}

The remaining cases cannot easily be dealt with analytically and so we use numerical techniques to determine the lower bound, $b_{f}^{\text {crit }}$, for which the wave moves in a positive direction. This critical value is found by numerically determining $f_{+}^{*}$ and $I\left(b_{f}, n_{1}\right)$ : a bisection method is employed to determine where the sign of $I\left(b_{f}, n_{1}\right)$ changes.

The results of Fig. 6 show that as $n_{1}$, the Hill coefficient controlling $\boldsymbol{F}$ production, increases the range of $b_{f}$ for which the wave travels in a positive direction expands to over ten times its value for $n_{1}=2$. Fig. 6(a) shows the window for small values of $n_{1}$ whilst Fig. 6(b) demonstrates the extent to which the window expands as $n_{1}$ becomes very large. These results show that in order to achieve a robust mechanism for gradient formation, in the sense described in Section 4.1.2, $n_{1}$ should be large. In the following, we will show that the presence of a second- 
a

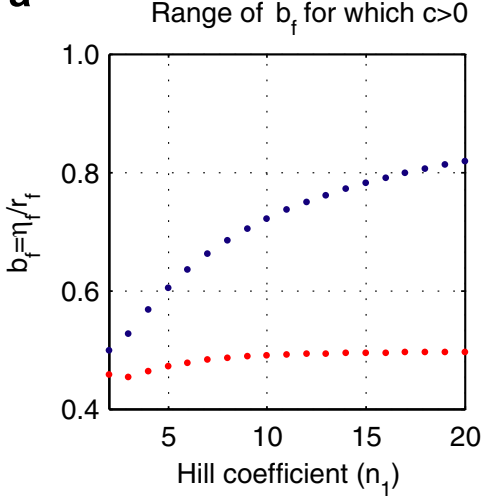

b

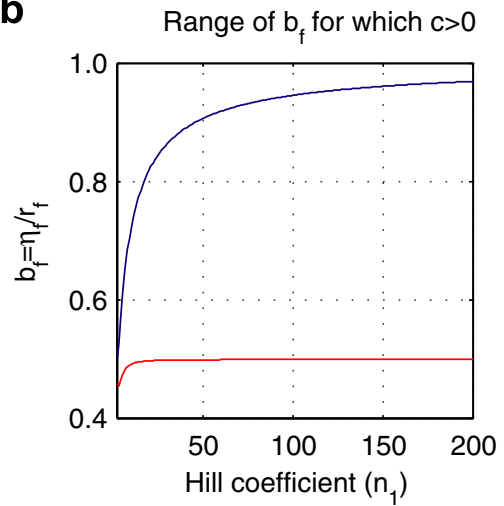

Fig. 6. The critical window for $b_{f}$ as $n_{1}$ increases: for values of $b_{f}$ between the dots/lines, the wave moves in a positive direction. The upper bound, $b_{f}^{\text {crit }}$, is the value of $b_{f}$ below which non-zero steady states exist and is given by Eq. (13): in (a) this is denoted by the blue dots and in (b) by the blue line. The lower bound, $b_{f}^{\text {crit }}$, is computed numerically by finding the value of $b_{f}$ for which $I\left(b_{f}, n_{1}\right)$ changes sign: in (a) this is denoted by the red dots and in (b) by the red line. Note that (b) is simply a re-drawing of (a) on enlarged axes. (For interpretation of the references to colour in this figure legend, the reader is referred to the web version of this article.)

ary morphogen, $\boldsymbol{A}$, can increase this window of $b_{f}$ parameter space, thereby relaxing the condition that $n_{1}$ be so large.

\subsection{Numerical solution}

Numerical solutions of the system are shown in Fig. 7 for two sets of parameter values. In both cases, the wave travels in a positive direction since the parameters are chosen within the window $\left(b_{f}^{\text {crit }_{l}}, b_{f}^{\text {crit }_{u}}\right)$. In general, numerical investigation shows that increasing $n_{1}$ increases the steady state level of $\boldsymbol{F}, f_{+}^{*}$, increases the slope of the wave and correspondingly decreases the wave speed. These changes in steady state and wave speed as a function of $b_{f}$ are shown in Fig. 8(a). The opposite effects are seen to occur with changes in $\eta_{f}$ and this is demonstrated in Fig. 8(b).

\section{The full model}

Armed with some insight into the problem we now return to the full model outlined in Section 3. As before, we investigate linear stability and the possibilities for travelling wavefronts, before using numerical techniques to determine the effects of a secondary morphogen. Before starting, we note that in Fig. 2 (which shows simulations of the full model) the parameters are such that $b_{f}=0.25$ which puts $b_{f}<b_{f}^{\text {crit }_{l}}$, yet the wave still moves in a positive direction. This already shows that the addition of a secondary morphogen widens the parameter space for which we obtain waves travelling in a positive direction, thereby increasing the robustness of the system. 

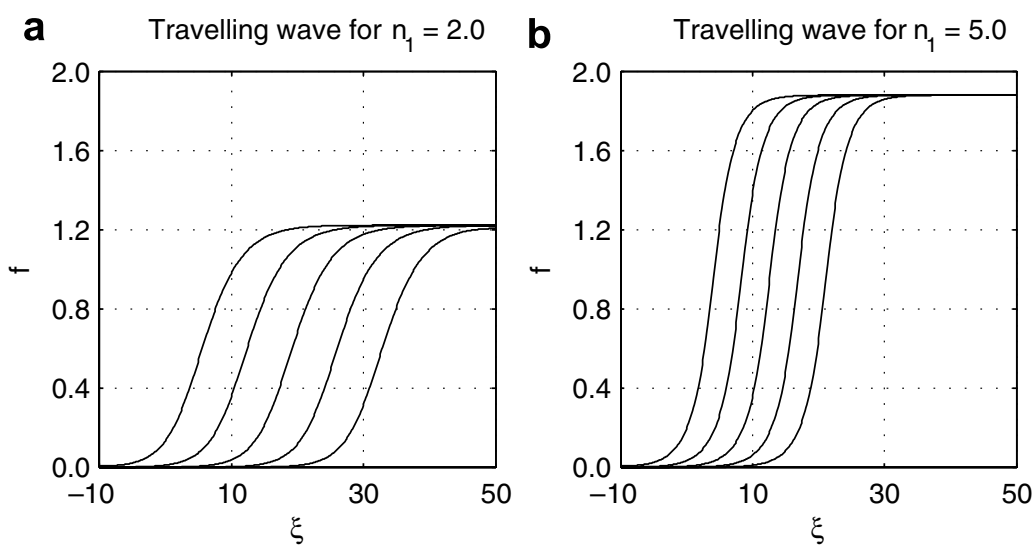

Fig. 7. Numerical solution of the 'knockout' case given by Eq. (10). (a) The Hill coefficient, $n_{1}$, is equal to two and $\eta_{f}=0.98$. (b) The Hill coefficient, $n_{1}$, is equal to five and $\eta_{f}=1.02$. In each case, the wave travels in a positive direction since the parameters are chosen within the window $\left(b_{f}^{\text {crit }_{l}}, b_{f}^{\text {crit }_{\mu}}\right)$, but the shape and speed of the wavefront changes with the model parameters. The plots are shown for $\tau=10,20,30,40,50$. Parameters are as follows: $r_{f}=2.0$ and $D=5.0$.
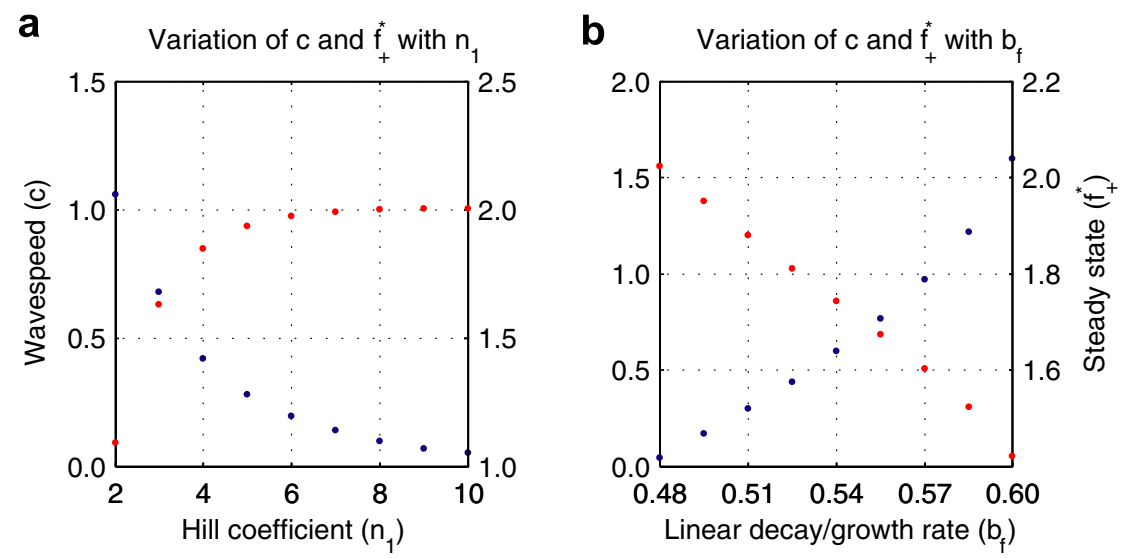

Fig. 8. The change in wave speed and $f_{+}^{*}$ as model parameters are varied. (a) Variation with Hill function coefficient $n_{1}$. (b) Variation with $b_{f}=\eta_{f} / r_{f}$. The blue dots show the wave speed and the red dots $f_{+}^{*}$. Parameters are as follows: (a) $b_{f}=0.498$; (b) $n_{1}=5$. (For interpretation of the references to colour in this figure legend, the reader is referred to the web version of this article.)

\subsection{Spatially uniform steady states}

For the general case specified by Eqs. (5) and (6) the null clines for $f$ are given by

$$
r_{f} \lambda_{f}^{n_{2}} f^{n_{1}}\left(\beta_{f}^{n_{3}}+a^{n_{3}}\right)-\left[\eta_{f}\left(\beta_{f}^{n_{3}}+a^{n_{3}}\right)+s_{f} a^{n_{3}}\right]\left(1+f^{n_{1}}\right)\left(\lambda_{f}^{n_{2}}+a^{n_{2}}\right) f=0 .
$$

Similarly, the null clines for $a$ are given by

$$
r_{a} \lambda_{a}^{n_{5}} a^{n_{4}}\left(\beta_{a}^{n_{6}}+f^{n_{6}}\right)-\left[\eta_{a}\left(\beta_{a}^{n_{6}}+f^{n_{6}}\right)+s_{a} f^{n_{6}}\right]\left(1+a^{n_{4}}\right)\left(\lambda_{a}^{n_{5}}+f^{n_{5}}\right) a=0 .
$$

As in the 'knockout' case, similar conditions are required for steady states of the form $\left(f^{*}, 0\right)$ and $\left(0, a^{*}\right)$ : 

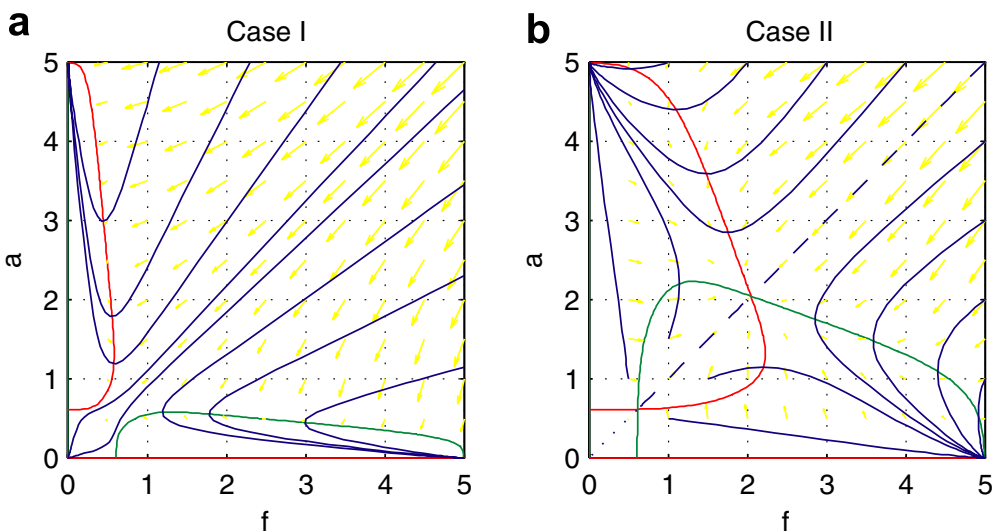

Fig. 9. Null clines for the general model specified by Eqs. (5) and (6). (a) Case I: there are no steady states with both $\boldsymbol{F}$ and $\boldsymbol{A}$ non-zero. (b) Case II: there are two steady states with both $\boldsymbol{F}$ and $\boldsymbol{A}$ non-zero. In both cases, the $\boldsymbol{F}$ null clines are shown in red and the $\boldsymbol{A}$ null clines in green. The velocity field is indicated by yellow arrows and the trajectories in blue. The broken lines in (b) denote the separatrix: trajectories with initial conditions on the dashed line go to the steady state with higher $\boldsymbol{F}$ and $\boldsymbol{A}$ whilst on the dotted line they tend to zero. Either side of the broken lines, the trajectories tend to the steady states $\left(f_{+}^{*}, 0\right)$ or $\left(0, a_{+}^{*}\right)$. Parameters are as follows: $r_{f}=1.5, r_{a}=1.5, \eta_{f}=0.3, \eta_{a}=0.3, s_{f}=0.1, s_{a}=0.1$, $\beta_{f}=3.0, \beta_{a}=3.0, n_{i}=4$ for $i=1, \ldots, 4, n_{i}=2$ for $i=5$, 6. Case I: $\lambda_{f}=0.5, \lambda_{a}=0.5$; Case II: $\lambda_{f}=2.0, \lambda_{a}=2.0$. (For interpretation of the references to colour in this figure legend, the reader is referred to the web version of this article.)

$$
b_{f}<b_{f}^{\mathrm{crit}_{u}}={\frac{\left(n_{1}-1\right)}{n_{1}}}^{\left(n_{1}-1\right) / n_{1}} \text { and } b_{a}<b_{a}^{\mathrm{crit}_{u}}={\frac{\left(n_{4}-1\right)}{n_{4}}}^{\left(n_{4}-1\right) / n_{4}},
$$

where $b_{f}=\eta_{f} / r_{f}$ and $b_{a}=\eta_{a} / r_{a}$. In all cases, we have the steady state $(f, a)=(0,0)$ and when the conditions above are satisfied we also have four further steady states: $(f, a)=\left(f_{ \pm}^{*}, 0\right)$ and $(f, a)=\left(0, a_{ \pm}^{*}\right)$.

Under the parameter conditions outlined above, there are two general possibilities for the phase plane, and null clines for these cases are plotted in Fig. 9, along with the velocity field and the trajectories. Case I, depicted in Fig. 9(a), shows the scenario in which there are no steady states with both $\boldsymbol{F}$ and $\boldsymbol{A}$ non-zero, whilst Case II, depicted in Fig. 9(b), shows the scenario in which there are two steady states with both $\boldsymbol{F}$ and $\boldsymbol{A}$ non-zero. In general, it is not possible to calculate these steady states explicitly, but after numerical examination of the phase plane we conclude that the parameters $\lambda_{f}$ and $\lambda_{a}$ play the greatest role in determining whether Case I or Case II arises: in Fig. 9, these are the only parameters which are changed between the two plots. For simplicity, we shall only consider Case I.

\subsection{Linear stability}

We linearise the system about the fixed points so that stability is given by the eigenvalues of the matrix $M_{l s}$ evaluated at the steady states, where

$$
M_{l s}=\left(\begin{array}{ll}
\frac{\partial p_{f}}{\partial f}-\frac{\partial d_{f}}{\partial f} & \frac{\partial p_{a}}{\partial f}-\frac{\partial d_{a}}{\partial f} \\
\frac{\partial p_{f}}{\partial a}-\frac{\partial d_{f}}{\partial a} & \frac{\partial p_{a}}{\partial a}-\frac{\partial d_{a}}{\partial a}
\end{array}\right)
$$


and the functions $p_{(.)}$and $d_{(.)}$are the non-dimensionalised production and decay terms. The linear stability of the five fixed points given above can be calculated as follows: $(f, a)=(0,0)$ is a stable node; $(f, a)=\left(f_{-}^{*}, 0\right)$ is a saddle point; $(f, a)=\left(f_{+}^{*}, 0\right)$ is a stable node; $(f, a)=\left(0, a_{-}^{*}\right)$ is a saddle point; $(f, a)=\left(0, a_{+}^{*}\right)$ is a stable node.

We can also determine the sign of the eigenvalues of the other two possible fixed points (when they exist) by considering the manner in which the null clines intersect. In doing so we see that: $(f, a)=\left(f_{-}^{\dagger}, a_{-}^{\dagger}\right)$ is an unstable point; $(f, a)=\left(f_{+}^{\dagger}, a_{+}^{\dagger}\right)$ is a saddle point. The stability of these points is demonstrated in Fig. 9 using the velocity field and trajectory plots.

\subsection{Travelling waves}

As mentioned above, we are interested in travelling waves which connect two stable steady states, one with high $f$ and low $a$ and the other with low $f$ and high $a$ : here these are the points $\left(f_{+}^{*}, 0\right)$ and $\left(0, a_{+}^{*}\right)$. We use the travelling wave ansatz $z=\xi-c \tau$ to transform Eqs. (5) and (6) to the following system of ordinary differential equations (ODEs):

$$
\begin{aligned}
& -c f^{\prime}=p_{f}(f, a)-d_{f}(f, a)+D f^{\prime \prime}, \\
& -c a^{\prime}=p_{a}(f, a)-d_{a}(f, a)+a^{\prime \prime},
\end{aligned}
$$

where $^{\prime}=\mathrm{d} / \mathrm{d} z$. This system can be converted to a first order system of ODEs by taking $f^{\prime}=g$ and $a^{\prime}=b$, and linear stability of each of the fixed points of the first order system can be determined by finding the roots of the equation

$$
\left[\lambda\left(\frac{c}{D}+\lambda\right)-M_{21}\right]\left[\lambda(c+\lambda)-M_{43}\right]-M_{23} M_{41}=0,
$$

where the coefficients $M_{i j}$ correspond to the entries of the matrix describing the first order ODE system linearised about the fixed point:

$$
\begin{aligned}
& M_{21}=\frac{1}{D}\left[\eta_{f}+\frac{s_{f} a^{n_{3}}}{\left(\beta_{f}^{n_{3}}+a^{n_{3}}\right)}-\frac{n_{1} r_{f} f^{n_{1}-1}}{\left(1+f^{n_{1}}\right)^{2}} \frac{\lambda_{f}^{n_{2}}}{\left(\lambda_{f}^{n_{2}}+a^{n_{2}}\right)}\right], \\
& M_{23}=\frac{1}{D}\left[\frac{n_{2} \lambda_{f}^{n_{2}} a^{n_{2}-1}}{\left(\lambda_{f}^{n_{2}}+a^{n_{2}}\right)^{2}} \frac{f^{n_{1}}}{\left(1+f^{n_{1}}\right)}+\frac{n_{3} s_{f} \beta_{f}^{n_{3}} a^{n_{3}-1} f}{\left(\beta_{f}^{n_{3}}+a^{n_{3}}\right)^{2}}\right], \\
& M_{41}=\left[\frac{n_{5} \lambda_{a}^{n_{5}} f^{n_{5}-1}}{\left(\lambda_{a}^{n_{5}}+f^{n_{5}}\right)^{2}} \frac{a^{n_{4}}}{\left(1+a^{n_{4}}\right)}+\frac{n_{6} s_{a} \beta_{a}^{n_{6}} f^{n_{6}-1} a}{\left(\beta_{a}^{n_{6}}+f^{n_{6}}\right)^{2}}\right], \\
& M_{43}=\left[\eta_{a}+\frac{s_{a} f^{n_{6}}}{\left(\beta_{a}^{n_{6}}+f^{n_{6}}\right)}-\frac{n_{4} r_{a} a^{n_{4}-1}}{\left(1+a^{n_{4}}\right)^{2}} \frac{\lambda_{a}^{n_{5}}}{\left(\lambda_{a}^{n_{5}}+f^{n_{5}}\right)}\right] .
\end{aligned}
$$

When the steady state has either $a$ or $f$ (or both) equal to zero then $M_{23} M_{41}=0$ and Eq. (26) can be solved to give the following solutions for $\lambda$ : 


$$
\lambda_{21}^{ \pm}=\frac{1}{2}\left[\frac{-c}{D} \pm \sqrt{\frac{c^{2}}{D^{2}}+4 M_{21}}\right] \text { and } \lambda_{43}^{ \pm}=\frac{1}{2}\left[-c \pm \sqrt{c^{2}+4 M_{43}}\right] .
$$

Hence both $(f, g, a, b)=\left(f_{+}^{*}, 0,0,0\right)$ and $(f, g, a, b)=\left(0,0, a_{+}^{*}, 0\right)$ have $\lambda_{21}^{-}, \lambda_{43}^{-}<0$ and $\lambda_{21}^{+}, \lambda_{43}^{+}>0$, and it is possible that there exists a trajectory in $(f, g, a, b)$ space connecting these two steady states. Since all the eigenvalues for these steady states are purely real, whatever the parameter values, we cannot establish from this analysis a minimum wave speed, only that the gradients of both $f$ and $a$ will be monotonic in $z$.

As before, we can determine the sign of the wave speed by multiplying Eqs. (24) and (25) by $f^{\prime}$ and $a^{\prime}$, respectively, and integrating with respect to $z$. Considering the case in which

$$
f(-\infty)=0, \quad f(\infty)=f_{+}^{*}, \quad a(-\infty)=0, \text { and } a(\infty)=a_{+}^{*},
$$

we see that $c$ must satisfy the following:

$$
\begin{aligned}
& c \int_{-\infty}^{\infty}\left(f^{\prime}\right)^{2} \mathrm{~d} z+\int_{0}^{f_{+}^{*}}\left[p_{f}(f, a)-d_{f}(f, a)\right] \mathrm{d} f=0, \\
& c \int_{-\infty}^{\infty}\left(a^{\prime}\right)^{2} \mathrm{~d} z-\int_{0}^{a_{+}^{*}}\left[p_{a}(f, a)-d_{a}(f, a)\right] \mathrm{d} a=0 .
\end{aligned}
$$

Hence the $c$ is positive (and the wave moves in a positive direction along the axis as $\tau$ increases) if

$$
I_{f}=\int_{0}^{f_{+}^{*}}\left[p_{f}(f, a)-d_{f}(f, a)\right] \mathrm{d} f<0,
$$

and vice versa for the integral, $I_{a}$, containing $p_{a}$ and $d_{a}$. First, we note that in the above equations, $f$ and $a$ are not independent of each other, so in order to compute the value of either $I_{f}$ or $I_{a}, a$ must be found as a function of $f$ or vice versa. Second, we also note that the expressions together form a 'consistency condition' for $f, a, f^{\prime}$ and $a$ '.

The first point above requires $I_{f}$ to be computed along a certain curve which lies within the region of $(f, a)$ space bounded by the lines $f=0, f_{+}^{*}$ and $a=0, a_{+}^{*}$. Supposing for now that $a$ is independent of $f$ then we can differentiate $I_{f}$ to get

$$
\frac{\partial I_{f}}{\partial a}=\int_{0}^{f_{+}^{*}}\left[\frac{\partial p_{f}}{\partial a}-\frac{\partial d_{f}}{\partial a}\right] \mathrm{d} f
$$

which gives

$$
\frac{\partial I_{f}}{\partial a}=\int_{0}^{f_{+}^{*}}\left[-\frac{n_{2} \lambda_{2}^{n_{2}} a^{n_{2}-1}}{\lambda_{f}^{n_{2}}+a^{n_{2}}} \frac{r_{f} f^{n_{1}}}{1+f^{n_{1}}}-\frac{n_{3} s_{f} \beta_{f}^{n_{3}} a^{n_{3}-1} f}{\beta_{f}^{n_{3}}+a^{n_{3}}}\right] \mathrm{d} f \leqslant 0 .
$$

So we see immediately that if $\left.I_{f}\right|_{a=0}<0$ (so that the wave travels in a positive direction in the 'knockout' case) then $I_{f}<0$ when $\boldsymbol{A}$ is present. Supposing that $\left.I_{f}\right|_{a=0}>0$, (so that the wave travels in a negative direction in the 'knockout' case) then since $\partial I_{f} / \partial a \leqslant 0$, it is possible that the introduction of $\boldsymbol{A}$ could cause the direction of travel to reverse. A necessary condition for this to occur is that 


$$
\left.I_{f}\right|_{a=a_{+}^{*}}=\int_{0}^{f_{+}^{*}}\left[p_{f}\left(f, a_{+}^{*}\right)-d_{f}\left(f, a_{+}^{*}\right)\right] \mathrm{d} f<0,
$$

and from the 'knockout' case we see that this occurs for $b_{f}^{\text {nec }}>b_{f}^{\mathrm{crit}_{l}}$ where

$$
b_{f}^{\text {nec }}=\frac{\eta_{f}+s_{f}^{+}}{r_{f} \lambda_{f}^{+}} \text {and } \lambda_{f}^{+}=\frac{\lambda_{f}^{n_{2}}}{\lambda_{f}^{n_{2}}+\left(a_{+}^{*}\right)^{n_{2}}}, \quad s_{f}^{+}=\frac{s_{f}\left(a_{+}^{*}\right)^{n_{3}}}{\beta_{f}^{n_{3}}+\left(a_{+}^{*}\right)^{n_{3}}} .
$$

In order to consider the integral along the actual path taken in $(f, a)$ space, we use a similar argument: we write

$$
I_{f}=\int_{0}^{f_{+}^{*}}\left[\frac{r_{f} \lambda_{f}^{a} f^{n_{1}}}{1+f^{n_{1}}}-\left(\eta_{f}+s_{f}^{a}\right) f\right] \mathrm{d} f
$$

where

$$
\lambda_{f}^{a}=\frac{\lambda_{f}^{n_{2}}}{\lambda_{f}^{n_{2}}+a^{n_{2}}} \in(0,1) \text { and } s_{f}^{a}=\frac{s_{f} a^{n_{3}}}{\beta_{f}^{n_{3}}+a^{n_{3}}} \in\left(0, s_{f}\right) \text { for } a>0 .
$$

Given the bounds on $\lambda_{f}^{a}$ and $s_{f}^{a}$ we deduce that the effective value of $b_{f}$ is increased:

$$
b_{f}^{\mathrm{eff}}=\frac{\eta_{f}+s_{f}^{a}}{r_{f} \lambda_{f}^{a}}>b_{f}=\frac{\eta_{f}}{r_{f}} .
$$

In other words, the addition of $\boldsymbol{A}$ increases the region of $\left(r_{f}, \eta_{f}\right)$ space in which the wave travels in a

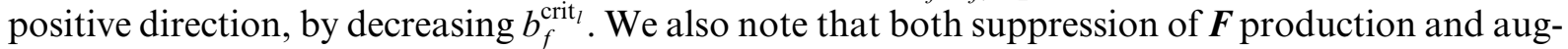
mented $\boldsymbol{F}$ decay by $\boldsymbol{A}$ have the same effect. In other words, the presence of a secondary morphogen increases the robustness of the primary morphogen gradient to changes in the system parameters, by widening the parameter space in which the wavefront travels in a positive $x$ direction.

Fig. 10 shows the actual change in wave speed as $\lambda_{f}$ and $s_{f}$ are varied. Fig. 10(a) demonstrates a decrease in wave speed as $\lambda_{f}$ is increased: a result of the fact that increasing $\lambda_{f}$ actually decreases
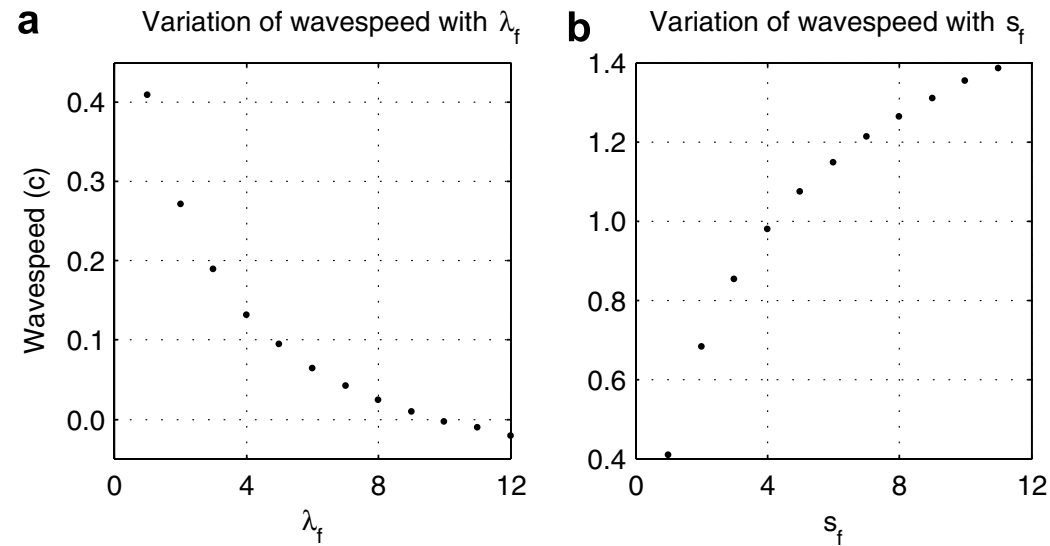

Fig. 10. The change in wave speed as model parameters are varied. (a) Variation with $\lambda_{f}$, the parameter affecting $\boldsymbol{F}$ production. (b) Variation with $s_{f}$, the parameter affecting $\boldsymbol{F}$ decay. Unless otherwise indicated on the graph, the parameter values are as in Fig. 2(a). 
the effect $\boldsymbol{A}$ has on the system. There are two things to note here: first, that for $\lambda_{f} \gtrsim 10$ the wave begins to travel in a negative direction; second, as $\lambda_{f}$ decreases (so that the effect of $a$ upon $f$ production increases) the variation in wave speed increases. Fig. 10(b) demonstrates the increase in wave speed as $s_{f}$ is increased: this can be understood by reasoning that increased decay of $\boldsymbol{F}$ leads to an increase in the rate of $\boldsymbol{A}$ production, so that $\boldsymbol{A}$ may advance along the axis more quickly. In this case, we see that the change in wave speed as $s_{f}$ varies becomes less marked as $s_{f}$ becomes large. It should be noted that although the plots in Fig. 10 are on different scales, our results suggest that a relatively high value of $s_{f}$ is preferable to a relatively low value of $\lambda_{f}$. In other words, increasing the rate of primary morphogen decay by the addition of a secondary morphogen seems to be a more robust mechanism than decreasing the rate of primary morphogen production by the addition of a secondary morphogen.

\section{Discussion}

In this work, we have investigated possible mechanisms by which two morphogens may interact in order to produce travelling wavefronts of expression along some developmental axis. The models constructed were based on a specific developmental context: the gradients of FGF8 and RA which exist along the AP axis of vertebrate embryos, controlling axis segmentation and neuronal differentiation. We started by constructing a generic model from basic experimental hypotheses, which we investigated both analytically and numerically. In order to gain insight into the model, and to compare with the case of a single morphogen, we first investigated a 'knockout' case in which the secondary morphogen, taken to be $\boldsymbol{A}$, was removed.

In the single morphogen case, we found a condition for the existence of travelling wave solutions and a further condition which must be satisfied by the model parameters in order to ensure that the wave of $\boldsymbol{F}$ regresses along the AP axis with time, i.e., that it moves in a positive direction. We showed that if production of the morphogen is controlled by Hill-type kinetics, a small Hill coefficient results in only a small window of parameter space that gives rise to regressing waves of $\boldsymbol{F}$. Increasing the Hill coefficient widens the parameter window, eventually to over ten times its original value, thereby making the system more robust to changes in parameter values, which may arise due to variations in morphogen production or decay, or from external factors.

Upon returning to the dual morphogen case, we were able to use these results to show that the presence of a secondary morphogen is able to widen the parameter space for which the waves regress along the axis. In this way, the two-morphogen model increases the robustness of the model to perturbation - it is more able to display the required behaviour as model parameters are varied.

Earlier in this work we described another aim: to elucidate the robustness of the wave speed to changes in parameter values. Both suppression of primary morphogen production and increase in primary morphogen decay by a secondary morphogen were show to increase the speed of the wavefront. However, it seems that as the effect of $a$ on $f$ decay increases, the change in the speed of the wavefront decreases, whilst increasing the effect of $a$ of $f$ production has the opposite effect. Therefore we postulate that the presence of a secondary morphogen, acting to increase the decay rate of a primary morphogen, will lead to a more robust mechanism for development, both in terms of an increased parameter space for a positive wave speed and the change in wave speed as the strength of the secondary morphogen increases. 
It is somewhat difficult to compare the results of our model to others which consider the role of secondary morphogens $[9,13]$. The reasons for this are as follows: first, our gradients are not stationary, second we consider their formation on an infinite, rather than a bounded domain, and third we consider a fairly generic system, rather than a specific developmental context. It is our aim that the knowledge and insight gained from this study will provide a foundation upon which more biologically accurate models may be built.

\subsection{Application to models for somite formation}

Several mathematical models for somite formation have been suggested in recent years, including one by the authors [41,42], which is based on the version of the 'Clock and Wavefront' model formulated by Pourquié and co-workers [6,36,37]. To date these models only consider a gradient of FGF8 along the AP axis: they are able to reproduce coherent somite formation and the anomalies observed experimentally upon local perturbation of the FGF8 gradient [6]. However, since RA is not explicitly modelled, they are not able to reproduce the experimental observations upon RA removal: the rate of AP axis extension is decreased and small somites form, although the total number of somites is regulated $[43,44]$. These facts are consistent with the results of this paper and the 'Clock and Wavefront' model which suggest that removal of RA would slow the progression of the FGF8 wavefront, thereby conferring the ability to segment upon fewer cells in each oscillation of the segmentation clock. One aim for future work is to integrate this system into these previous models for somite formation $[41,42]$ to see if it is indeed capable of mimicking the results.

\section{Conclusion}

In summary, the model suggests that introduction of a secondary morphogen, acting in an antagonistic manner to a primary morphogen, is able to increase robustness of the primary morphogen gradient to perturbation and that it also plays a role in determining wave speed and shape. The models and context considered here are excellent paradigms for other scenarios in which morphogen gradients are known to act and as such, these results have direct application to many other developmental systems.

\section{Acknowledgments}

R.E.B. wishes thank Lloyds Tercentenary Foundation for a Lloyds Tercentenary Foundation Fellowship, Research Councils UK for an RCUK Academic Fellowship in Mathematical Biology, St. Hugh's College, Oxford for a Junior Research Fellowship and the Stowers Institute for Medical Research, Kansas City for a visiting position.

\section{References}

[1] T. Tabata, Y. Takei, Morphogens, their identification and regulation, Development 131 (2004) 703.

[2] L. Wolpert, One hundred years of positional information, Trends Genet. 12 (1996) 359. 
[3] J. Briscoe, J. Ericson, Specification of neuronal fates in the ventral neural tube, Curr. Opin. Neurobiol. 11 (2001) 43.

[4] D. Morisato, K.V. Anderson, Signalling pathways that establish the dorso-ventral pattern of the Drosophila embryo, Annu. Rev. Genet. 29 (1995) 371.

[5] C. Tickle, Morphogen gradients in vertebrate limb development, Cell Dev. Biol. 10 (1999) 345.

[6] J. Dubrulle, M.J. McGrew, O. Pourquié, FGF signalling controls somite boundary position and regulates segmentation clock control of spatiotemporal Hox gene activation, Cell 106 (2001) 219.

[7] T.-X. Jiang, H.-S. Jung, R.B. Widelitz, C.-M. Chuong, Self-organization of periodic patterns by dissociated feather mesenchymal cells and the regulation of size, number and spacing of primordia, Development 126 (1999) 4997.

[8] R. Diez del Corral, K. Storey, Opposing FGF and retinoid pathways: a signalling switch that controls differentiation and patterning onset in the extending vertebrate body axis, Bioessays 26 (2004) 869.

[9] B. Houchmandzadeh, E. Wieschaus, S. Leibler, Precise domain specification in the developing Drosophila embryo, Phys. Rev. E 72 (6) (2005) 061920.

[10] B. Houchmandzadeh, E. Wieschaus, S. Leibler, Establishment of developmental precision and proportions in the early Drosophila embryo, Nature 415 (2002) 798.

[11] A.D. Lander, Q. Nie, F.Y.M. Wan, Spatially distributed morphogen production and morphogen gradient formation, Math. Biosci. Eng. 2 (2005) 239.

[12] T. Bollenbach, K. Kruse, P. Pantazis, M. González-Gaitán, F. Jülicher, Robust formation of morphogen gradients, Phys. Rev. Lett. 94 (1) (2005), 018103-1-018103-4.

[13] T. Aegerter-Wilmsen, C. Aegerter, T. Bisseling, Model for the robust establishment of precise proportions in the early Drosophila embryo, J. Theor. Biol. 234 (1) (2005) 13.

[14] M. Howard, P. Rein ten Wolde, Finding the center reliably: robust patterns of developmental gene expression, Phy. Rev. Lett. 95 (2006), 208103-1-208103-4.

[15] J. Dubrulle, O. Pourquié, Coupling segmentation to axis formation, Development 131 (2004) 5783.

[16] P.H. Crossley, S. Martinez, G.R. Martin, Midbrain development induced by FGF8 in the chick embryo, Nature 380 (1996) 66.

[17] V. Dupé, A. Lumsden, Hindbrain patterning involves graded responses to retinoic acid signalling, Development 128 (2001) 2199.

[18] M. Ensini, T.N. Tsuchida, H.G. Belting, T.M. Jessell, The control of rostrocaudal pattern in the developing spinal chord: specification of motor neuron subtype identity is initiated by signals from paraxial mesoderm, Development 125 (1998) 969.

[19] A. Gould, N. Itasaki, R. Krumlauf, Initiation of rhombomeric Hoxb4 expression requires induction by somites and a retinoid pathway, Neuron 21 (1998) 39.

[20] N. Itasaki, J. Sharpe, A. Morrison, R. Krumlauf, Reprogramming Hox expression in the vertebrate hindbain: influence of paraxial mesoderm and rhombomere transposition, Neuron 16 (1996) 487.

[21] A. Linville, E. Gumusaneli, R.A.S. Chandraratna, T.F. Schilling, Independent roles for retinoic acid in segmentation and neuronal differentiation in the zebrafish hindbrain, Dev. Biol. 270 (2004) 186.

[22] J.P. Liu, E. Laufer, T.M. Jessell, Assigning the positional identity of spinal motor neurons: rostrocaudal patterning of Hox-c expression by FGFs, Gdf11 and retinoids, Neuron 32 (2001) 997.

[23] J. Muhr, E. Graziano, S. Wilson, T.M. Jessell, T. Edlund, Convergent inductive signals specify midbrain, hindbrain and spinal chord identity in gastrula stage chick embryos, Neuron 23 (1999) 689.

[24] R. Diez del Corral, I. Olivera-Martinez, A. Goriely, E. Gale, M. Maden, K. Storey, Opposing FGF and retinoid pathways control ventral neural pattern, neuronal differentiation and segmentation during body axis extension, Neuron 40 (2003) 65.

[25] B.G. Novitch, H. Wichterle, T.M. Jessell, S. Sockanathan, A requirement for retinoic acid-mediated transcriptional activation in ventral neural patterning and motor neuron specification, Neuron 40 (2003) 81.

[26] L. Wilson, F. Gale, D. Chambers, M. Maden, Retinoic acid and the control of dorsoventral patterning in the avian spinal chord, Dev. Biol. 269 (2004) 433.

[27] C.D. Stern, S.E. Fraser, R.J. Keynes, D.R.N. Primmett, A cell lineage analysis of segmentation in the chick embryo, Development 104S (1988) 231.

[28] O. Pourquié, The segmentation clock: converting embryonic time into spatial pattern, Science 301 (2003) 328.

[29] A. Gossler, M. Hrabě de Angelis, Somitogenesis, Curr. Top. Dev. Biol. 38 (1998) 225. 
[30] H.L. Stickney, M.S.J. Barresi, S.H. Devoto, Somite development in zebrafish, Dev. Dyn. 219 (2000) 287.

[31] F.E. Stockdale, W.J. Nikovits, B. Christ, Molecular and cellular biology of avian somite development, Dev. Dyn. 219 (2000) 304.

[32] J.R. Collier, D. McInerney, S. Schnell, P.K. Maini, D.J. Gavaghan, P. Houston, C.D. Stern, A cell cycle model for somitogenesis: mathematical formulation and numerical solution, J. Theor. Biol. 207 (2000) 305.

[33] S. Schnell, P.K. Maini, D. McInerney, D.J. Gavaghan, P. Houston, Models for pattern formation in somitogenesis: a marriage of cellular and molecular biology, C.R. Biologies 325 (2002) 179.

[34] J. Cooke, E.C. Zeeman, A clock and wavefront model for control of the number of repeated structures during animal morphogenesis, J. Theor. Biol. 58 (1976) 455.

[35] I. Palmeirim, D. Henrique, D. Ish-Horowicz, O. Pourquié, Avian hairy gene expression identifies a molecular clock linked to vertebrate segmentation and somitogenesis, Cell 91 (1997) 639.

[36] J. Dubrulle, O. Pourquié, From head to tail: links between the segmentation clock and antero-posterior patterning of the embryo, Curr. Opin. Gen. Dev. 5 (2002) 519.

[37] O. Pourquié, The chick embryo: a leading model for model in somitogenesis studies, Mech. Dev. 121 (2004) 1069.

[38] A.D. Lander, Q. Nie, F.Y.M. Wan, Do morphogen gradients arise by diffusion? Dev. Cell 2 (2002) 785.

[39] P.C. Fife, J.B. McLeod, The approach of solutions of nonlinear diffusion equations to travelling wave solutions, Arch. Rat. Mech. Anal. 65 (1977) 335.

[40] J.D. Murray, Mathematical biology I: an introduction, 3rd ed., vol. I, Springer, 2003.

[41] R.E. Baker, S. Schnell, P.K. Maini, A clock and wavefront mechanism for somite formation, Dev. Biol. 293 (2006) 116.

[42] R.E. Baker, S. Schnell, P.K. Maini, A mathematical investigation of a clock and wavefront model for somitogenesis, J. Math. Biol. 52 (4) (2006) 458.

[43] M. Maden, A. Graham, M. Zile, E. Gale, Abnormalities of somite development in the absence of retinoic acid, Int. J. Dev. Biol. 44 (2000) 151.

[44] K. Niederreither, V. Subbarayan, P. Dollé, P. Chambon, Embryonic retinoic acid synthesis is essential for early mouse post-implantation development, Nat. Genet. 21 (1999) 444. 